\title{
Thermonuclear explosions of rapidly rotating white dwarfs
}

\section{Deflagrations}

\author{
J. M. M. Pfannes ${ }^{1}$, J. C. Niemeyer ${ }^{1,2}$, W. Schmidt ${ }^{1,2}$, and C. Klingenberg ${ }^{3}$ \\ 1 Lehrstuhl für Astronomie, Universität Würzburg, Am Hubland, 97074 Würzburg, Germany \\ e-mail: pfannes@astro.uni-wuerzburg.de \\ 2 Institut für Astrophysik, Universität Göttingen, Friedrich-Hund-Platz 1, 37077 Göttingen, Germany \\ e-mail: [niemeyer;schmidt] @astro.physik.uni-goettingen.de \\ 3 Institut für Angewandte Mathematik, Universität Würzburg, Am Hubland, 97074 Würzburg, Germany \\ e-mail: klingenberg@mathematik.uni-wuerzburg.de
}

Received 10 March 2009 / Accepted 27 October 2009

\section{ABSTRACT}

\begin{abstract}
Context. Turbulent deflagrations of Chandrasekhar mass white dwarfs are commonly used to model type Ia supernova explosions. In this context, rapid rotation of the progenitor star is plausible but has so far been neglected.

Aims. The aim of this work is to explore the influence of rapid rotation on the deflagration scenario.

Methods. We use three dimensional hydrodynamical simulations to model turbulent deflagrations ignited within a variety of rapidly rotating CO WDs obeying rotation laws suggested by accretion studies.

Results. We find that rotation has a significant impact on the explosion. The flame develops a strong anisotropy with a preferred direction towards the stellar poles, leaving great amounts of unburnt matter along the equatorial plane.

Conclusions. The large amount of unburnt matter is contrary to observed spectral features of SNe Ia. Thus, rapid rotation of the progenitor star and the deflagration scenario are incompatible in order to explain SNe Ia.
\end{abstract}

Key words. supernovae: general - hydrodynamics - methods: numerical

\section{Introduction}

Driven by the outstanding potential of type Ia supernovae (SNe Ia) to reconstruct the expansion history of the late universe, a number of observational campaigns have collected a wealth of empirical data about the lightcurves, spectra, and host populations of these powerful events (Wood-Vasey et al. 2007; Riess et al. 2007). This progress is in contrast to the sobering fact that despite many decades of theoretical and computational efforts, we are still lacking a solid understanding of the explosion mechanism, or the combination of different mechanisms, that can explain the class of $\mathrm{SNe}$ Ia in its entirety. While the majority of SN Ia explosions is believed to involve the rapid thermonuclear combustion of Chandrasekhar mass $\mathrm{CO}$ white dwarfs (WDs) (Hillebrandt \& Niemeyer 2000; Röpke 2008), it is still debated exactly how and where the star ignites (Woosley et al. 2004; Schmidt \& Niemeyer 2006; Röpke et al. 2006), and whether the combustion front propagates subsonically as a deflagration or supersonically as a detonation (Plewa et al. 2004; Röpke et al. 2007a; Röpke \& Niemeyer 2007). However, it is clear that if the front is subsonic, its effective propagation rate is governed only by the turbulence produced by large-scale instabilities (Niemeyer \& Woosley 1997).

From a purely theoretical point of view, the turbulent deflagration model is favoured compared with its competitors that invoke a spontaneous transition to a detonation (Niemeyer 1999), but large 3D simulations indicate it may be incapable to explain the full class of SNe Ia (Röpke et al. 2007a). Nevertheless, because of its conceptual simplicity and lack of free parameters, we will use it as a testbed for studying the influence of rapid rotation on the outcome of the explosion. Since the duration of thermonuclear burning is determined by the dynamical time scale of the WD, the star must rotate nearly critically in order for rotation to have any significant impact. This appears plausible at first sight, since most progenitor scenarios for SNe Ia involve the accretion of almost a solar mass of material from a binary companion before the thermonuclear runaway, allowing the WD to pick up a substantial amount of angular momentum. Indeed, some evolutionary calculations predict critical rotation of the WD at the time of the explosion (Yoon \& Langer 2005). On the other hand, the transport and dissipation of angular momentum inside the star are poorly understood and direct observations are absent. Hence, we treat the amplitude and shape of the rotation law as essentially undetermined and attempt to investigate their influence in a parameterised manner. As we will show, our general conclusion is robust with respect to the details of the rotation law: nearly critical rotation of the progenitor WD together with a pure deflagration model for the combustion front are inconsistent with the observations of SNe Ia.

The first multidimensional simulations of rapidly rotating exploding WDs were carried out by Steinmetz et al. (1992). However, in contrast to our work they concentrated on pure detonations instead of deflagrations. We will revisit the prompt detonation scenario in a follow-up paper.

This paper is structured as follows. Section 2 summarises the theoretical background on rotation of SNe Ia progenitor stars. The motivation of the employed rotation laws is given. Different methods for the numerical initiation of the burning process are presented in Sect. 3. Section 4 describes the computational grid, the flame modelling, treatment of the gravitational potential, the 
employed WD models, numerical stability, and the parameter study. The results of the numerical study are listed in Sect. 5. The impact of rotation on the explosion is explained by considering buoyancy effects. Also, the influence of model parameters and shear motion caused by rotation is presented. An interpretation with respect to spectral features finalises the section. Section 6 concludes the paper.

\section{Rotation of $\mathrm{CO}$ white dwarfs}

In the context of the single degenerate progenitor scenario for SNe Ia (Livio 2000), a white dwarf star consisting of carbon and oxygen (CO WD) accretes matter from a non-degenerate companion via a Keplerian disc. Since the average WD mass is $\sim 0.6 M_{\odot}$, the amount of matter that has to be accreted prior to $\mathrm{C}$ ignition is large (this occurs soonest for non-rotating $\mathrm{CO}$ WDs close to their Chandrasekhar mass, i.e. at $M_{\mathrm{WD}} \sim 1.4 M_{\odot}$ ). In addition to the matter itself, angular momentum is accreted. Therefore it is reasonable to assume that the WD spins up during its accretion phase.

Yoon \& Langer (2004) (YL) analyse the physics of accreting white dwarfs (AWDs) in detail and claim that in case of rapid rotation, the limiting mass for $\mathrm{C}$ ignition or collapse can greatly exceed the value of the Chandrasekhar-mass (Yoon \& Langer 2005). YL's stellar evolution code includes the effects of accretion-induced heating and energy transport, angular momentum transport by various instabilities and the effect of rotation on the WD structure by modifications in the momentum and energy conservation equations. Electron and ion viscosities, relevant for the onset of instabilities, are taken into account. Transport of the accreted angular momentum is treated as a diffusion process.

As their main conclusion regarding the angular velocity profile of the SN Ia progenitor, accreting WDs rotate differentially throughout their evolution. The angular velocity reaches a maximum, $\Omega_{\text {peak }}$, within the star, increasing from the centre outwards but decreasing toward the surface. This behaviour is attributed to the fact that the slowly rotating inner part contracts faster than the rapidly rotating surface layers as the total mass of the accreting star increases. Its value is in the range of $1.5 \mathrm{rad} / \mathrm{s} \lesssim \Omega_{\text {peak }} \lesssim$ $6.5 \mathrm{rad} / \mathrm{s}$ when the central density of the WD reaches the ignition density of $2.0 \times 10^{9} \mathrm{~g} / \mathrm{cm}^{3}$. The corresponding total masses are $\sim 1.5$ and $\sim 2.1 M_{\odot}$, respectively.

As YL's results suggest, the rotation law(s) realised by nature may depend on different factors such as efficiency of accreted angular momentum transport, the accretion time scale, the time scale for loss of angular momentum, the total binary mass budget, or magnetic fields. Therefore, in this work we study the impact of a parameterised class of rotators with different rotation laws and, consequently, different explosion masses. Specifically, four types of AWD rotators are considered as SN Ia progenitors. They are - listed in order of increasing stellar mass - denoted by AWD1, AWD2, AWD4, and AWD3 and include the full spread of AWD masses Physical quantities and numerical details are itemised in Table 1 . The central density is $\rho_{\mathrm{c}}=2.0 \times 10^{9} \mathrm{~g} / \mathrm{cm}^{3}$ for all models. $M_{\text {high }}$ is the mass for all material if $\rho>5.248 \times 10^{7} \mathrm{~g} / \mathrm{cm}^{3}$, and $M_{\text {med }}$ if $5.248 \times 10^{7} \mathrm{~g} / \mathrm{cm}^{3}>$ $\rho>1.047 \times 10^{7} \mathrm{~g} / \mathrm{cm}^{3} . \beta$ denotes the ratio of rotational energy and gravitational binding energy, $J$ the angular momentum. In the non-rotating case $\left(\rho_{\mathrm{c}}=2.0 \times 10^{9} \mathrm{~g} / \mathrm{cm}^{3}, M=1.4 M_{\odot}\right) M_{\text {high }}$ and $M_{\text {med }}$ are $1.268 M_{\odot}(90.6 \%)$ and $1.110 M_{\odot}(7.9 \%)$, respectively. Furthermore, the non-rotating star possesses the following quantities: $r=2.1905 \times 10^{8} \mathrm{~cm}, E_{\mathrm{int}}=2.5228 \times 10^{51} \mathrm{erg}$, $E_{\text {grav }}=-3.0275 \times 10^{51} \mathrm{erg}, E_{\text {rot }}=0.000 \times 10^{50} \mathrm{erg}$, and
Table 1. Physical quantities and numerical coefficients of the constructed AWD initial models.

\begin{tabular}{rcccc}
\hline \hline Model & AWD1 & AWD2 & AWD4 & AWD3 \\
\hline$\Omega_{\mathrm{c}}[\mathrm{rad} / \mathrm{s}]$ & 1.5999 & 4.6074 & 4.5934 & 3.9847 \\
\hline$c_{1}$ & 7.3470 & -12.065 & -16.265 & -18.823 \\
$c_{2}$ & 62.006 & 136.61 & 162.39 & 196.62 \\
$c_{3}$ & -275.25 & -436.83 & -480.84 & -563.38 \\
$c_{4}$ & 342.62 & 509.56 & 531.85 & 600.25 \\
$c_{5}$ & -137.09 & -200.88 & -200.81 & -218.51 \\
$\bar{s}_{\text {rigid }}$ & 0 & 0 & 0.2 & 0 \\
$r_{\text {equator }} / r_{\text {pol }}$ & 1.629 & 1.710 & 1.796 & 2.183 \\
$\Omega_{\text {peak }}[\mathrm{rad} / \mathrm{s}]$ & 4.4126 & 4.5158 & 5.1986 & 5.2236 \\
$M\left[M_{\odot}\right]$ & 1.6374 & 1.7428 & 1.7911 & 2.0150 \\
$M_{\text {high }}\left[M_{\odot}\right]$ & 1.393 & 1.481 & 1.490 & 1.469 \\
$M_{\text {high }} / M[\%]$ & 85.1 & 85.0 & 83.2 & 72.9 \\
$M_{\text {med }}\left[M_{\odot}\right]$ & 0.180 & 0.200 & 0.227 & 0.401 \\
$M_{\text {med }} / M[\%]$ & 11.0 & 11.5 & 12.7 & 19.9 \\
$r_{\text {pol }}\left[10^{8} \mathrm{~cm}\right]$ & 2.0180 & 1.8985 & 1.8833 & 1.8836 \\
$r_{\text {equator }}\left[10^{8} \mathrm{~cm}\right]$ & 3.2871 & 3.2471 & 3.3822 & 4.1116 \\
$E_{\text {int }}\left[10^{51} \mathrm{erg}\right]$ & 2.7395 & 2.8895 & 2.9148 & 2.9604 \\
$E_{\text {grav }}\left[10^{51} \mathrm{erg}\right]$ & -3.6701 & -4.0330 & -4.1419 & -4.5283 \\
$E_{\text {rot }}\left[10^{50} \mathrm{erg}\right]$ & 1.748 & 2.625 & 2.991 & 4.465 \\
$E_{\text {bind }}\left[10^{50} \mathrm{erg}\right]$ & -7.558 & -8.810 & -9.280 & -11.214 \\
$\beta[\%]$ & 4.7639 & 6.5092 & 7.2206 & 9.8608 \\
$J\left[10^{50} \mathrm{~g} \mathrm{~cm}{ }^{2} / \mathrm{s}\right]$ & 0.91512 & 1.1968 & 1.3602 & 2.1951 \\
\hline
\end{tabular}

$E_{\text {bind }} \equiv E_{\text {int }}+E_{\text {grav }}+E_{\text {rot }}=-5.047 \times 10^{50}$ erg. Since rigid rotation is believed to occur in the pre-supernova convective core due to very efficient exchange of angular momentum, rigid rotation of the interior is assumed for the AWD4 model (see Fig. 1) while the rotators AWD1 to 3 rotate differentially everywhere.

Besides AWD rotation, different rotation laws have been tested for pre-supernova WDs. If there are strong braking magnetic torques or efficient viscous angular momentum transport, a critically rotating rigid rotator might result. In contrast to differentially rotating WDs, the mass of this kind of object increases only slightly by $0.07 M_{\odot}$ for a central density $\rho_{\mathrm{c}}=$ $2.0 \times 10^{9} \mathrm{~g} / \mathrm{cm}^{3}$. In addition, the frequently used $j-$ const. and $v$ - const. rotation laws, denoting approximately constant specific angular momentum and rotational velocity, respectively, are employed (cf. Table 2). The parameter $a d r$ denotes the fraction of the equatorial radius up to which rotation has approximately constant specific angular momentum and rotational velocity, respectively (Eriguchi \& Müller 1985). For both of these rotation laws, angular velocity decreases steadily from the rotational axis outwards. See Sect. 4 for details of the numerical treatment of the employed rotating WDs.

Under certain restrictions, it is also possible to interpret our model in the context of the double-degenerate scenario for $\mathrm{SNe} \mathrm{Ia}$, i.e. the merger and immediate explosion of two WDs. Rapid rotation is particularly well motivated in this case. However, two conditions need to be satisfied for our model assumptions to apply: first, in order to reach hydrostatic equilibrium of the initial configuration, the explosion must only be ignited after a sufficiently long delay of several dynamical time scales. Second, it is unclear whether in this scenario, ignition will occur first near the surface and propagate inward (see Hillebrandt \& Niemeyer 2000 for details and references). Note that while the former is simply a restriction on the applicability of our initial conditions, the latter is a serious problem for the double-degenerate scenario in itself. For the purpose of our study, we remain agnostic about the progenitor history as long as the conditions for viability of our initial model are met. 
J. M. M. Pfannes et al.: Deflagrations of rapidly rotating white dwarfs. I.
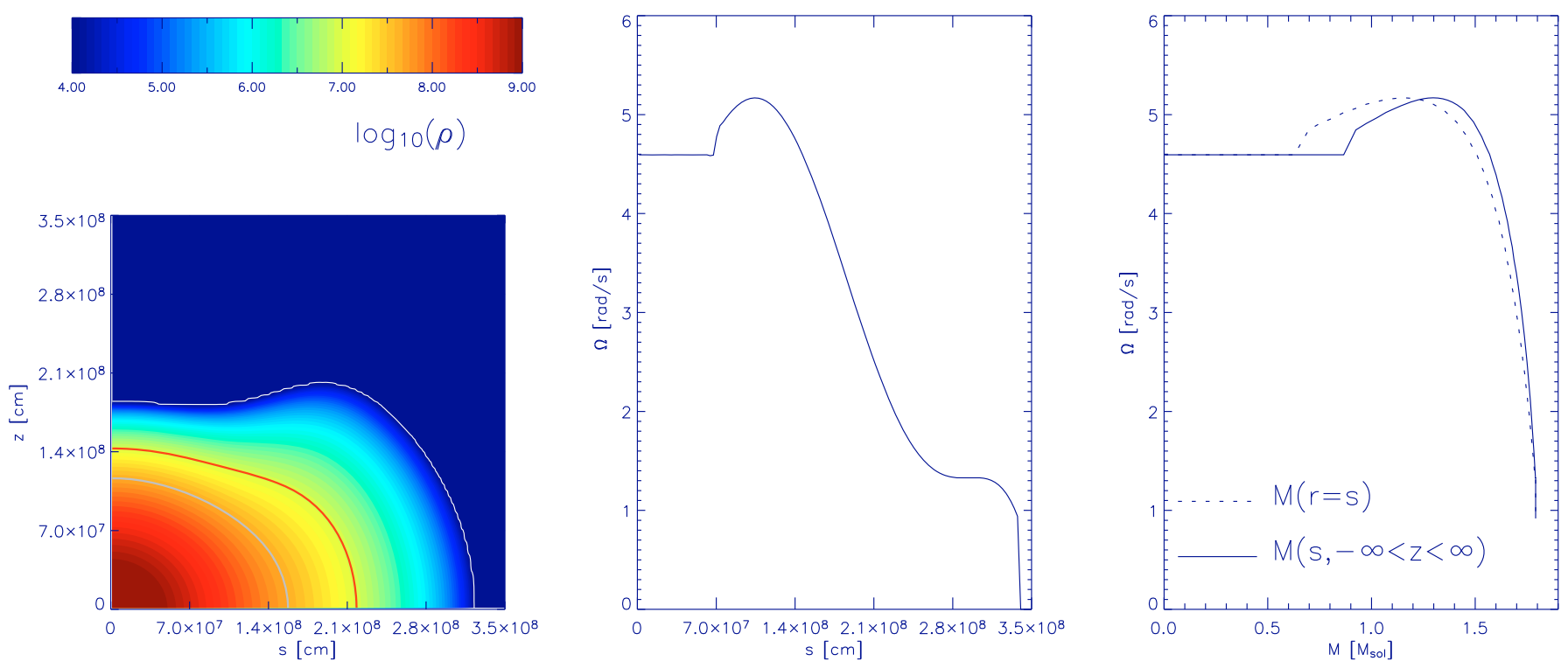

Fig. 1. Density contour lines (left panel) and the rotation law both in spatial (middle panel) and mass coordinates (right panel) for the AWD4 model. Unlike the dotted line that denotes the spherical mass coordinate, the solid line line indicates the cylindrical mass coordinate in order to describe cylindrical rotation. Note that the extent of the convective core (i.e., the rigidly rotating inner part) is estimated following Yoon \& Langer (2004).

Table 2. The same parameters as in Table 1 for rigid rotation and the "exotic" $j_{\text {const }}$ and $v_{\text {const }}$ rotators.

\begin{tabular}{rccc}
\hline \hline Model & Rigid & $j_{\text {const }}$ & $v_{\text {const }}$ \\
\hline$\Omega_{\mathrm{c}}[\mathrm{rad} / \mathrm{s}]$ & 0.37242 & 6.4076 & 10.120 \\
\hline$r_{\text {equator }} / r_{\text {pol }}$ & 1.477 & 2.183 & 2.183 \\
$a d r$ & - & 0.5 & 0.2 \\
$M\left[M_{\odot}\right]$ & 1.4661 & 1.8025 & 1.7067 \\
$M_{\text {high }}\left[M_{\odot}\right]$ & 1.302 & 1.534 & 1.493 \\
$M_{\text {high }} / M[\%]$ & 88.8 & 85.1 & 87.5 \\
$M_{\text {med }}\left[M_{\odot}\right]$ & 0.126 & 0.191 & 0.157 \\
$M_{\text {med }} / M[\%]$ & 8.6 & 10.6 & 9.2 \\
$r_{\text {pol }}\left[10^{8} \mathrm{~cm}\right]$ & 2.1721 & 1.8087 & 1.8134 \\
$r_{\text {equator }}\left[10^{8} \mathrm{~cm}\right]$ & 3.2092 & 3.9481 & 3.95858 \\
$E_{\text {int }}\left[10^{51} \mathrm{erg}\right]$ & 2.5878 & 3.0072 & 2.9678 \\
$E_{\text {grav }}\left[10^{51} \mathrm{erg}\right]$ & -3.2074 & -4.2779 & -4.0604 \\
$E_{\text {rot }}\left[10^{50} \mathrm{erg}\right]$ & 0.442 & 3.163 & 2.393 \\
$E_{\text {bind }}\left[10^{50} \mathrm{erg}\right]$ & -5.754 & -9.544 & -8.533 \\
$\beta[\%]$ & 1.3769 & 7.3941 & 5.8933 \\
$J\left[10^{50} \mathrm{~g} \mathrm{~cm}{ }^{2} / \mathrm{s}\right]$ & 0.37747 & 1.3785 & 1.0535 \\
\hline & & &
\end{tabular}

\section{Ignition conditions}

The explosion is assumed to be a turbulent deflagration (Hillebrandt \& Niemeyer 2000). The structure of the flame surface at the time of ignition is known to have a strong impact on the explosion outcome. In order to investigate the impact of certain ignition situations, we used a variety of different methods to initiate the burning front in our simulations.

A standard flame morphology that has been employed in several applications is the $c 3$ configuration (Reinecke et al. 2002), representing a central ignition with three bulges in azimuthal direction per octant. Besides the fact that the $c 3$ ignition is reproducible and ensures similar conditions even if initiated in different initial models, a major advantage of the $c 3$ ignition is the applicability for coarse numerical resolutions.

As a modification of the static multi-point ignition Röpke (2005), the stochastic ignition model initiates flame seeds randomly at different instants in time (Schmidt \& Niemeyer 2006). The probability of an ignition depends on the background temperature and the magnitude of the convective temperature fluctuations which are modelled by a mixing-length approach (Woosley et al. 2004; Wunsch \& Woosley 2004). Thus, the density of the emerging ignitions decreases with the distance from the centre and becomes statistically symmetric in angular directions. Due to the expansion of the white dwarf, the ignition probability decreases with time. The only parameter of the model, the exponentiation parameter $C_{\mathrm{e}}$, controls the overall speed of the process and thus the total number of ignition events.

An alternative scenario is the dipole jet flow (Woosley et al. 2004), where ignition sites are clustered along the rotation axis in one of the two hemispheres. The alignment of ignitions with the outward flow direction is explained by the fact that the condition necessary for ignition is met after stellar material has been transported through the hot stellar centre by the dipole jet flow. However, the dipole flow - also likely to occur in the nonrotating WD as a consequence of the convective pre-supernova motion - might be eliminated by a moderate amount of rotation (Kuhlen et al. 2006).

\section{Numerical method}

We use the hydrodynamics code PROMETHEUS (Reinecke 2001) for the simulation of explosions of rotating WDs in three dimensions. The computational grid is a moving grid composed of two nested sub-grids (Röpke 2005). The inner grid captures the burned region and the flame surface, while the outer grid expands together with the expanding WD. A crucial parameter for numerical simulations of SNe Ia is flame propagation velocity, because the evolution of the flame front and, consequently, the energy release from thermonuclear burning is greatly enhanced by turbulence. Following Schmidt et al. (2006a) and Schmidt et al. (2006b), the speed of the flame front is computed by a subgrid scale model that is based on a balance law for the turbulent energy. 
While previous simulations were performed with a gravitational potential that is spherically symmetric, we employ a multipole solver for the computation of the gravitational potential of rotating stars. A parameter study concerning different expansion contributions in rotating WDs showed that $\Phi_{g}(\boldsymbol{r})$ is approximated with sufficient accuracy if the axisymmetric multipole expansion is truncated after the quadrupole term. The numerical implementation was adapted from the public FLASH code (Fryxell et al. 2000). The FLASH implementation of the multipole solver is in turn adapted from the original implementation by Müller \& Steinmetz (1995).

Rotating density stratifications in hydrostatic equilibrium had to be generated as initial conditions for the hydrodynamic simulations (see Fig. 1 for an example). We constructed initial models using the method of Eriguchi \& Müller (1985). This method, which is an iterative procedure of correcting the density field based on local deviations from hydrostatic equilibrium, guarantees the creation of stars in hydrostatic equlilibria for a broad range of rotation laws. The WD equation of state was approximated using the piecewise polytropic approximation method (Müller \& Eriguchi 1985). Moreover, the rotators obey the characteristic AWD rotation laws as shown by Yoon $\&$ Langer (2004). This was achieved with the implementation of a polynomial representation of the angular velocity (which was terminated after the 5th order; cf. Table 1 for the chosen coefficients):

$\Omega(s)= \begin{cases}\Omega_{\mathrm{c}} & \text { for } 0 \leq \bar{s} \leq \bar{s}_{\text {rigid }} \\ \Omega_{\mathrm{c}}+c_{1} \bar{s}+c_{2} \bar{s}^{2} & \\ +c_{3} \bar{s}^{3}+c_{4} \bar{s}^{4}+c_{5} \bar{s}^{5} & \text { for } \bar{s}_{\text {rigid }}<\bar{s} \leq \bar{s}_{\max }\end{cases}$

Here, $\bar{s}$ is the normalised distance from the rotation axis and $\bar{s}_{\text {rigid }}$ the normalised distance from the rotation axis out to the position for which the angular velocity has a constant value of $\Omega_{\mathrm{c}}$ :

$\bar{s} \equiv \frac{s}{r_{\text {surf }}^{\text {equator }}} \quad$ and $\quad \bar{s}_{\text {rigid }} \equiv \frac{s_{\text {rigid }}}{r_{\text {surf }}^{\text {equator }}}$.

Since a strong magnetic field might prevent the rapid rotation of accreting WD's (Yoon \& Langer 2004), and the possible shape of the rotation law may vary to a large degree for different assumptions in the accretion model or simply from star to star, the initial models employed for this study were chosen to cover a broad range of possible rotation laws and, accordingly, masses. Besides the AWD rotation laws, we also considered rigid rotation as well as the $j-$ const. and $v-$ const. rotation laws, both stating decreasing angular velocity outwards (cf. Eriguchi \& Müller 1985, for details on both rotation laws).

Tables 1 and 2 summarise the parameters of our models. The masses of the AWD initial models range from $1.64 M_{\odot}$ to $2.07 M_{\odot}$. Model AWD4 contains a rigidly rotating inner core (up to the equatorial position $s=6.76 \times 10^{6} \mathrm{~cm}$ ) that is thought to arise from the pre-supernova convective motion within the star. The rotation law for this model is plotted in Fig. 1. Note that differential rotators can become heavier than the critical rigid rotator since $\Omega$ is allowed to have a high value within the star and to drop towards the equatorial surface. As a consequence, overcritical rotation is avoided.

The rotation law was not fitted for an exact overlap with those proposed by YL. In contrast, our parametrisation was chosen to emulate the central angular velocity, $\Omega_{\mathrm{c}}$, the maximum of $\Omega$, and the total mass. Given the uncertainties in the predictions for rotation laws, this choice is sufficiently realistic for our purposes.
The mass accretion from a Keplerian disc implies a critically rotating surface of the AWD rotators which cannot easily be incorporated because the initial model algorithm aborts when mass shedding for a model is detected. Therefore it is assumed that the surfaces of most constructed models in this study do not rotate critically. This is not problematic since the outer layers of a star do not affect the nuclear burning in the interior.

We tested whether the initial models remain hydrodynamically stable on the grid structure used for the SNe Ia simulations. Hydrodynamical simulations were followed to $t=1.5 \mathrm{~s}$, the time when burning would have stopped if the WD had been ignited. A comparison of density contours at different instants served as a simple check for hydrostatic stability. Up to $t=1.0 \mathrm{~s}$, the contour lines retained their shape except at the surface. At later times, the WD's core density also decreases somewhat because of the limited feasibility to perform circular motion on a rectangular cartesian grid. Two reasons are responsible for amplified deviations in the outer layers. First, the resolution of the grid is lower outside the core region and second, the pressure gradient is neglected below the threshold density of $\rho=10^{-3} \mathrm{~g} / \mathrm{cm}^{3}$. A more quantitative test was done by looking at the radial velocities emerging during the test of hydrostatic stability. Radial velocities in the inner region are at all instants found to be $\lesssim 4 \times 10^{7} \mathrm{~cm} / \mathrm{s}$ and therefore one order of magnitude lower than the characteristic velocities after ignition. In conclusion, stability of all discretised models on the grid can be assumed.

In order to cover a broad range of possible explosion scenarios, we varied the following parameters:

- the initial model: norot, rigid, AWD1, AWD2, AWD4, AWD3, $j_{\text {const }}$, and $v_{\text {const }}$

- the ignition scenario:

- static ignition (c3);

- stochastic ignition; here, the ignition speed was varied by changing the parameter $C_{\mathrm{e}}$ (cf. Sect. 3).

All simulations for this work were carried out in three spatial dimensions. At least one hemisphere or even a full star (necessary for the simulation of the dipole jet flow ignition) were considered. Since our objective was a parameter study, we chose a relatively coarse initial resolution of $7.5 \times 10^{5} \mathrm{~cm}$ on a $256^{2} \times 128$ grid for one hemisphere. In order to get an estimate of the accuracy of all simulations, the "rigid $C_{\mathrm{e}}=5 \times 10^{4}$ " explosion model was redone with doubled resolution. It was in remarkable agreement with the run on a normal grid (the explosion energetics differ by less than $4 \%$, the produced species differ by less than $2 \%$ ). This result indicates that the resolution employed for most of the simulations is sufficient since the explosion energetics and species composition are almost identical.

\section{Results}

In the following, we will first analyse deflagration for the AWD4 rotator, which is ignited by stochastic ignition. The strong impact of rotation on the flame morphology can be regarded as typical for the deflagration of rapidly rotating WDs. Variations of the stochastic ignition process and the influence of the rotation laws are investigated next. We will also comment on the role of shear motion and the expected spectral features.

\subsection{Anisotropic burning induced by rotation}

The existence of a preferred direction for the flame propagation in rotating white dwarfs can be attributed to two effects. First, the 


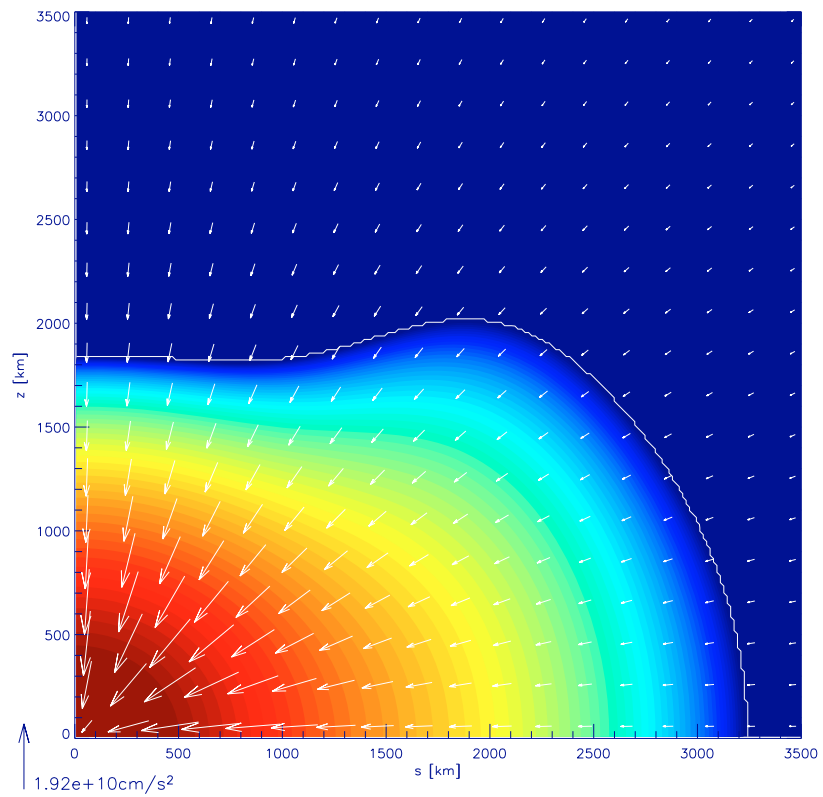

(a) $g_{\text {grav }}$

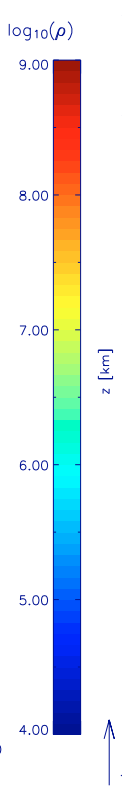

$1.92 \mathrm{e}+10 \mathrm{~cm} / \mathrm{s}^{2}$

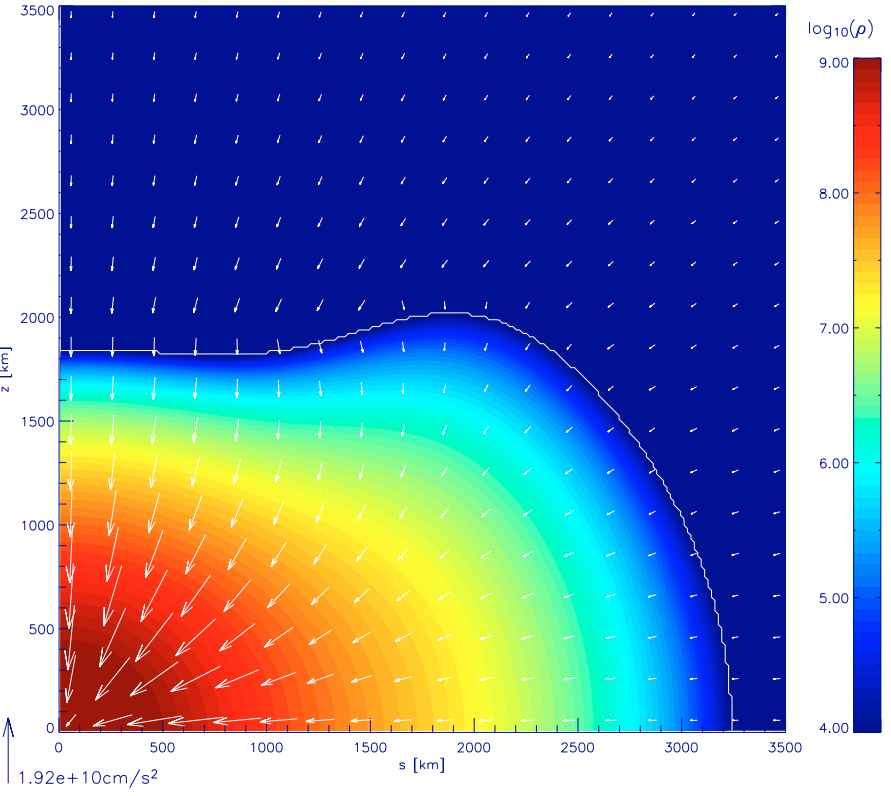

(b) $g_{\text {eff }}$

Fig. 2. Density contour lines together with the gravitational acceleration $\boldsymbol{g}_{\text {grav }} \mathbf{a}$ ) and the effective gravitational acceleration $\left.\boldsymbol{g}_{\text {eff }} \mathbf{b}\right)$ that is indicated by arrows, respectively, for the AWD4 rotator. Note that $\boldsymbol{g}_{\text {eff }}$ is orthogonal to the coinciding isobaric and isopycnic surfaces (outside the star, the centrifugal acceleration is zero).

ashes of SNe Ia are subject to buoyant motion, which is stronger in polar direction. Second, for convective motion in equatorial direction, the burning products have to gain angular momentum, whereas the opposite is true for inflowing fuel. This effectively gives rise to an angular momentum barrier, which suppresses the turbulent spreading of the deflagration front in the direction orthogonal to the rotation axis.

Figure 2(a) shows the gravitational acceleration $\boldsymbol{g}_{\text {grav }}$ inside the AWD4 rotator. As a result of the larger density gradient along the rotation axis, $\boldsymbol{g}_{\text {grav }}$ is stronger (indicated by longer arrays) in this direction compared to the equatorial plane. Buoyancy is caused by the effective gravitational acceleration $\boldsymbol{g}_{\text {eff }}$, i.e., the gravitational acceleration including the centrifugal acceleration as plotted in Fig. 2(b). $g_{\text {eff }}$ is orthogonal to the coinciding isobaric and isopycnic surfaces and exactly compensates the pressure gradient in hydrostatic equilibrium. The difference in $\boldsymbol{g}_{\text {eff }}$ along the polar and equatorial direction is even larger than the difference in $\boldsymbol{g}_{\text {grav }}$, which can be seen in Fig. 3 .

For the velocity $v_{\mathrm{b}}$ of a buoyant bubble, the following expression holds (Davies \& Taylor 1950; Glimm \& Li 1988; Read 1984):

$v_{\mathrm{b}}=C_{1} \sqrt{\frac{1}{2} \operatorname{Atg} g_{\mathrm{eff}} D}$,

where the dimensionless constant $C_{1}$ is about $0.5, D$ is the bubble diameter, and the Atwood number At $=\left(\rho_{\mathrm{f}}-\rho_{\mathrm{b}}\right) /\left(\rho_{\mathrm{f}}+\rho_{\mathrm{b}}\right)$ specifies the density contrast between the rising burning products of density $\rho_{\mathrm{b}}$ in fuel of density $\rho_{\mathrm{f}}>\rho_{\mathrm{b}}$. The dependence of $v_{\mathrm{b}}$ on the position inside the star is mainly given by the variation of $\boldsymbol{g}_{\text {eff }}$. The magnitude of $\boldsymbol{g}_{\text {eff }}$ differs by a factor up to 1.3 in polar and equatorial directions. (see Fig. 3). From Eq. (3) follows that the rising velocity of ashes in polar direction can exceed the velocity in equatorial direction by $15 \%$. The velocity of the fastest bubbles is of the order $\sim 10^{8} \mathrm{~cm} / \mathrm{s}$ (with At $=0.5$,

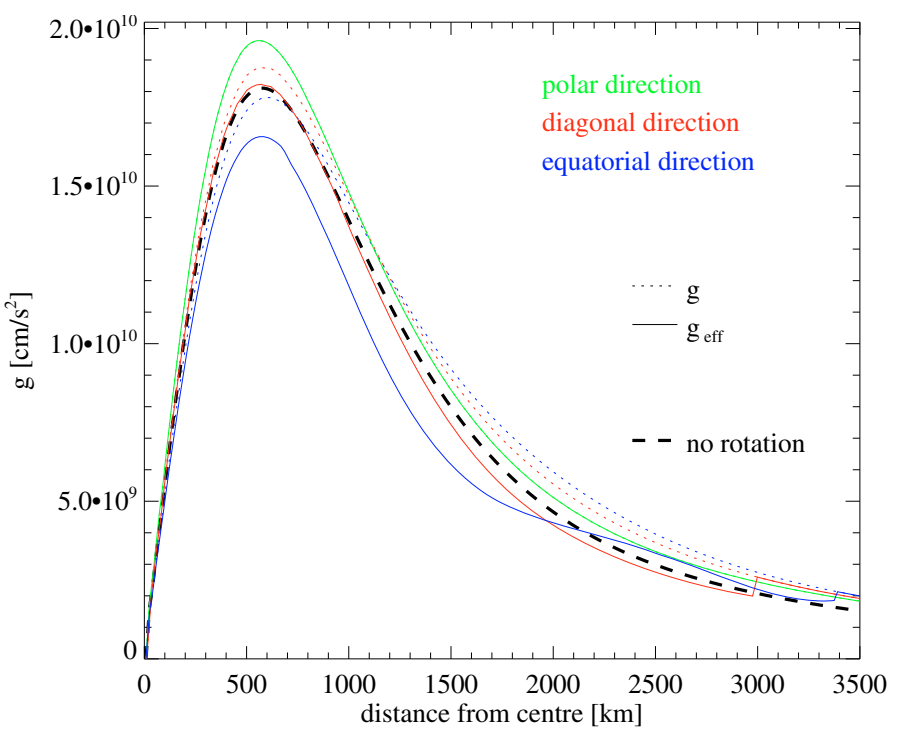

Fig. 3. Gravitational acceleration $\boldsymbol{g}_{\text {grav }}$ (dotted line) and the effective gravitational acceleration $\boldsymbol{g}_{\text {eff }}$ (solid line) along the rotation axis, the stellar diagonal, and the equatorial plane for the AWD4 rotator. The edges of the red line (at $\sim 3000 \mathrm{~km}$ ) and the blue line (at $\sim 3400 \mathrm{~km}$ ) appear due to the absence of centrifugal acceleration outside the star. The dashed thick black line represents the spherically symmetric gravitational acceleration for the non-rotating star of equal central density.

$\left|\boldsymbol{g}_{\text {eff }}\right|=2 \times 10^{10} \mathrm{~cm} / \mathrm{s}^{2}$, and a bubble diameter of $10^{8} \mathrm{~cm}$ ), a value that is approximately $10 \%$ of the speed of sound. Accordingly, smaller bubbles rise more slowly. A lower limit for the rising speed is of the order of $10^{6} \mathrm{~cm} / \mathrm{s}$ (with At $=0.1$, a lower gravitational acceleration and the bubble dimension of $10^{5} \mathrm{~cm}$ ). 

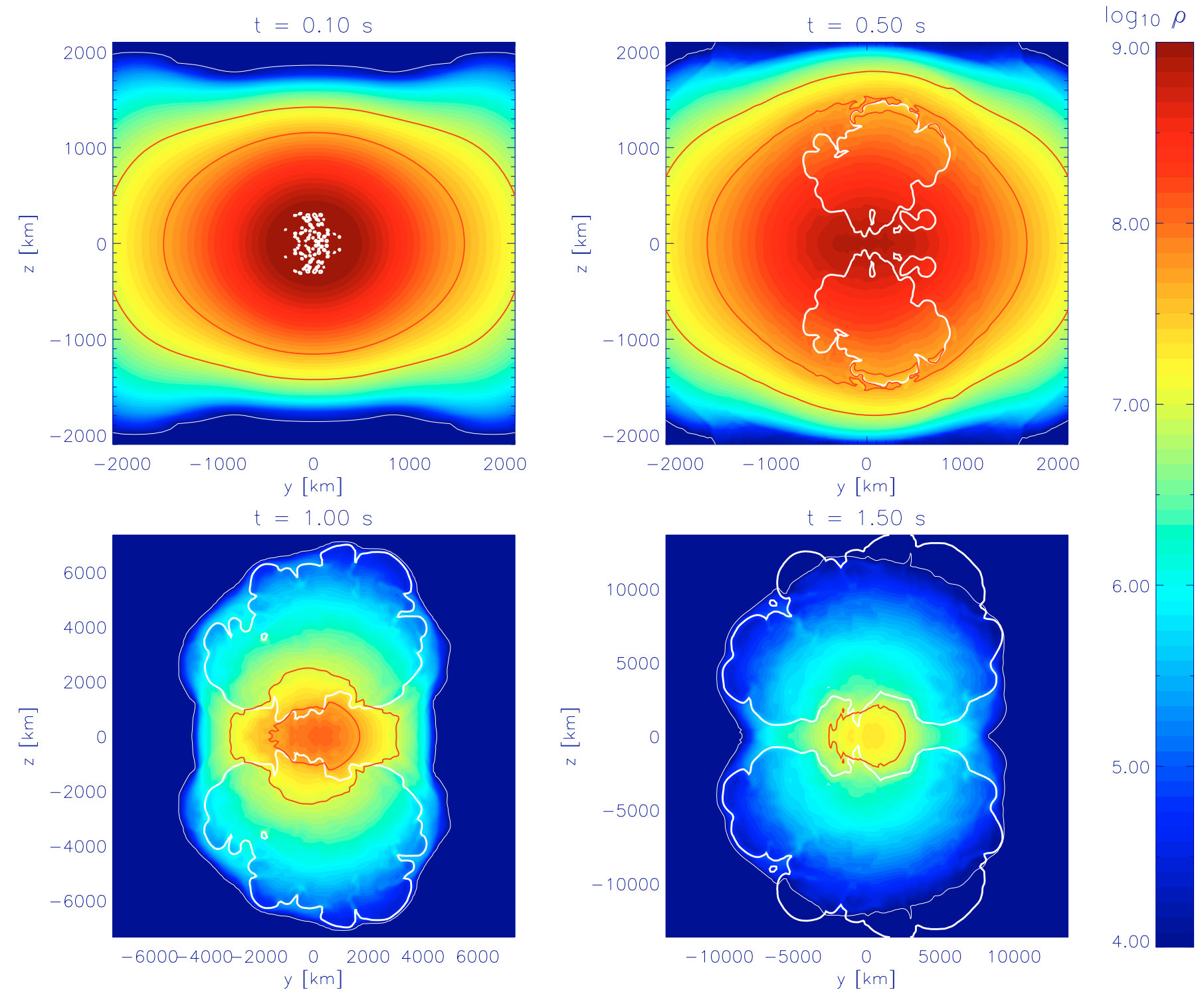

Fig. 4. Density contour lines for the pure deflagration of the AWD4 rotator at different instants for stochastic ignition $\left(C_{\mathrm{e}}=5 \times 10^{4}\right)$. Cross sections along the rotation axis of these simulations exhibiting equatorial symmetry are shown.

In the following, we consider the explosion of the AWD4 rotator in the stochastic ignition scenario with $C_{\mathrm{e}}=5 \times 10^{4}$. Figure 4 shows the flame surface (represented by thick white contours) embedded in density profiles at four instants. The simulation was carried out for one hemisphere only. Thus the contour plots - cross sections in the $y z$-plane along the rotation axis - exhibit equatorial symmetry. During the first $0.1 \mathrm{~s}, 363$ ignition spots are set isotropically in the interior of the star. Even at this early period, an agglomeration of the flame around the rotation axis (which points along the $x$ axis) is visible. Subsequently, the anisotropy of the flame grows because of the effects discussed above. In addition, the turbulent flame propagation speed is amplified in the polar direction due to the stronger production of turbulence. The thin contour lines (red in the online version) in Fig. 4 enclose densities greater than $5.248 \times 10^{7} \mathrm{~g} / \mathrm{cm}^{3}$ (threshold for producing iron group elements) and $1.047 \times 10^{7} \mathrm{~g} / \mathrm{cm}^{3}$ (threshold for producing intermediate mass elements).

In conclusion, both the buoyancy that is enhanced at the poles in rotating WDs and the blocked mixing orthogonal to the rotation axis lead to a strongly anisotropic flame that leaves behind unburnt material at the stellar centre.

\subsection{Ignition speed in the stochastic ignition scenario}

As outlined in Sect. 3, the stochastic ignition process contains one free parameter: the exponentiation parameter $C_{\mathrm{e}}$ that controls the overall speed of the creation of ignitions. Just as in the original work by Schmidt \& Niemeyer (2006), this parameter was varied over a broad range in order to study the influence of $C_{\mathrm{e}}$. For this reason, the AWD3 rotator was ignited for $C_{\mathrm{e}}$ between $5 \times 10^{1}$ and $5 \times 10^{7}$ (see Table 3) in the isotropic mode, i.e., for ignitions that are placed without preference. $\Omega$ denotes the total spherical angle covered by the simulation domain.

As summarised in Table 3, increasing $C_{\mathrm{e}}$ results in a higher amount of iron group elements (IGEs), while the production of intermediate mass elements (IMEs) does not change significantly. However, similar to the results obtained by Schmidt \& Niemeyer (2006), the energy output and the IGE production do not increase further for $C_{\mathrm{e}}>5 \times 10^{5}$. This effect can be 
J. M. M. Pfannes et al.: Deflagrations of rapidly rotating white dwarfs. I.

Table 3. Energetics and compositions for the deflagration models activated by stochastic ignition with different values for $C_{\mathrm{e}}$ in the AWD3 rotator $\left(M_{\mathrm{tot}}=2.02 M_{\odot}\right)$.

\begin{tabular}{|c|c|c|c|c|c|c|}
\hline$C_{\mathrm{e}}$ & $5 \times 10^{1}$ & $5 \times 10^{3}$ & $5 \times 10^{4}$ & $5 \times 10^{5}$ & $5 \times 10^{7}$ & $10^{4}$ \\
\hline Mode & Iso & Iso & Iso & Iso & Iso & Dipole \\
\hline$\Omega[\pi]$ & 2 & 2 & 2 & 2 & 2 & 4 \\
\hline$I_{2 \pi}$ & 13 & 152 & 391 & 809 & 19980 & 247 \\
\hline \multicolumn{7}{|l|}{$t=5 \mathrm{~s}$} \\
\hline$E_{\text {kin }}\left[10^{50} \mathrm{erg}\right]$ & 2.87 & 3.61 & 5.30 & 6.90 & 6.11 & 4.43 \\
\hline$E_{\text {tot }}\left[10^{50} \mathrm{erg}\right]$ & 0.21 & 1.33 & 3.54 & 5.47 & 4.62 & 2.55 \\
\hline$E_{\text {nuc }}\left[10^{51} \mathrm{erg}\right]$ & 1.050 & 1.182 & 1.420 & 1.619 & 1.535 & 1.310 \\
\hline IGEs $\left[M_{\odot}\right]$ & 0.40 & 0.51 & 0.69 & 0.79 & 0.74 & 0.62 \\
\hline$\left[\begin{array}{ll}\% & M_{\mathrm{tot}}\end{array}\right]$ & 20 & 25 & 34 & 39 & 37 & 31 \\
\hline IMEs $\left[M_{\odot}\right]$ & 0.51 & 0.46 & 0.41 & 0.46 & 0.45 & 0.40 \\
\hline$\left[\begin{array}{lll}\% & M_{\mathrm{tot}}\end{array}\right]$ & 25 & 23 & 20 & 23 & 23 & 20 \\
\hline $\mathrm{C}+\mathrm{O}\left[M_{\odot}\right]$ & 1.10 & 1.04 & 0.91 & 0.76 & 0.82 & 0.99 \\
\hline$\left[\begin{array}{ll}\% & M_{\mathrm{tot}}\end{array}\right]$ & 55 & 52 & 46 & 38 & 40 & 49 \\
\hline
\end{tabular}

explained by the saturation or even decrease of the flame surface during the ignition phase for an extremely higher number of ignition events (see Röpke et al. 2006, for a discussion of the same trend for the static multi-spot ignition). However, employing the value $C_{\mathrm{e}}=5 \times 10^{5}$ amounts to burning the entire core almost instantaneously because within the first tenth of a second almost $10^{3}$ ignitions are initiated within a central region of the typical radius of $\sim 350 \mathrm{~km}$. Since high values of $C_{\mathrm{e}}$ not only seem unphysical but also cannot serve as a remedy for the problems arising for the stochastic ignition in combination with rotation (cf. Sect. 5.3.2), the values of $C_{\mathrm{e}}=5 \times 10^{3}$ and $C_{\mathrm{e}}=5 \times 10^{4}$ were used in this work.

The dipole jet flow scenario was also tested by means of the AWD3 rotator. However, as the impact of buoyancy already suggests (cf. Sect. 5.1), this ignition realisation does not ameliorate the problem of remaining fuel close to the centre. If ignitions are set mainly in one hemisphere (without loss of generality, the southern one), the northern hemisphere is also burnt at a later time, but $\mathrm{C}$ and $\mathrm{O}$ are left over in the core. In the non-rotating case, a strong off-centre ignition also leads to a delayed burning of the distant stellar part, but less centrally located fuel remains (Röpke et al. 2007b).

We also found that the detailed location of randomly chosen ignition points has a significant impact on the outcome of the simulation. The "rigid $C_{\mathrm{e}}=5 \times 10^{3}$ " explosion model was simulated twice and is summarised in the two colums rigid and rigid ${ }^{*}$ of Table 5 . The only difference was a different seed for the random number generator which sets the ignition position within a shell whose number is determined by the Poisson process. Due to the randomness of the ignition process, the total number of ignitions is not entirely but approximately the same: 167 vs. 163 ignitions were set during the simulations. However, since bubbles inserted close to the rotation axis grow much more rapidly and tend to dominate the explosion, the generated nuclear energy differs by $\sim 20 \%$ (the more fuel remains unburnt, the lower is the value of produced nuclear energy). This demonstrates the high sensitivity of the rotating WD deflagration scenario on initial conditions. The sensitivity to the initial distribution of the seed flame was also observed for non-rotating WDs but in that context it caused only small deviations (Schmidt \& Niemeyer 2006). Any stellar rotation enhances this finding as a result of the oblate shape of the rotators.
Table 4. Energetics and compositions for the deflagration model with $c 3$ ignition in different rotators.

\begin{tabular}{rcccccc}
\hline \hline & Norot & Rigid & AWD1 & AWD2 & AWD4 & AWD3 \\
\hline$M_{\text {tot }}\left[M_{\odot}\right]$ & 1.40 & 1.47 & 1.64 & 1.74 & 1.79 & 2.02 \\
$\Omega[\pi]$ & 2 & 2 & 2 & 2 & 2 & 2 \\
\hline$t=0 \mathrm{~s}$ & \multicolumn{7}{c}{} \\
$E_{\text {grav }}\left[10^{51} \mathrm{erg}\right]$ & -3.027 & -3.204 & -3.665 & -4.027 & -4.135 & -4.518 \\
$E_{\text {rot }}\left[10^{50} \mathrm{erg}\right]$ & 0.00 & 0.44 & 1.75 & 2.62 & 2.99 & 4.46 \\
$\beta[\%]$ & 0.00 & 1.38 & 4.78 & 6.50 & 7.22 & 9.87 \\
\hline$t=5 \mathrm{~s}$ & & & & & & \\
$E_{\text {kin }}\left[10^{50} \mathrm{erg}\right]$ & 6.49 & 6.53 & 6.63 & 5.99 & 5.42 & 5.28 \\
$E_{\text {tot }}\left[10^{50} \mathrm{erg}\right]$ & 5.89 & 5.87 & 5.74 & 4.84 & 4.09 & 3.50 \\
$E_{\text {nuc }}\left[10^{51} \mathrm{erg}\right]$ & 1.076 & 1.129 & 1.291 & 1.318 & 1.289 & 1.419 \\
$\mathrm{IGEs}\left[M_{\odot}\right]$ & 0.54 & 0.56 & 0.65 & 0.65 & 0.64 & 0.67 \\
{$\left[\% M_{\text {tot }}\right]$} & 38 & 39 & 40 & 37 & 35 & 33 \\
$\mathrm{IMEs}\left[M_{\odot}\right]$ & 0.28 & 0.30 & 0.34 & 0.36 & 0.35 & 0.44 \\
{$\left[\% M_{\text {tot }}\right]$} & 20 & 20 & 20 & 21 & 20 & 22 \\
$\mathrm{C}+\mathrm{O}\left[M_{\odot}\right]$ & 0.58 & 0.61 & 0.65 & 0.73 & 0.80 & 0.90 \\
{$\left[\% M_{\text {tot }}\right]$} & 42 & 41 & 40 & 42 & 45 & 45 \\
\hline
\end{tabular}

\subsection{Variation of the rotation law}

Differences in the rotation law give rise to substantial variations of the total mass of the thermonuclear supernova progenitor. In particular, rapidly rotating WDs can greatly exceed the canonical Chandrasekhar mass: the progenitor stars under consideration possess masses in the range of $1.40 M_{\odot}$ (no rotation) and $2.02 M_{\odot}$ (model AWD3). An important conclusion can already be drawn by looking at the mass densities which the different WD models exhibit. The total mass increases by $\sim 44 \%$ from the non-rotating star to the AWD3 rotator. However, the mass fraction with densities greater than $5.25 \times 10^{7} \mathrm{~g} / \mathrm{cm}^{3}$, for which IGEs are produced, grows by only $\sim 16 \%$. The reason is that the fraction of dense material within the star decreases from $\sim 91 \%$ in the non-rotating star to only $\sim 73 \%$ in the AWD3 rotator which - although heavier by $\sim 0.6 M_{\odot}-$ contains only $\sim 0.2 M_{\odot}$ more IGEs. This is illustrated in Figs. 5(a), 5(b). Therefore it is doubtful whether the pure deflagration of rapidly rotating superChandrasekhar-mass models yields significantly more ${ }^{56} \mathrm{Ni} \mathrm{com-}$ pared to the non-rotating case. We consider the different rotation laws for the $c 3$ and stochastic ignition scenarios in turn.

\subsection{1. c3 ignition}

Figure 7(a) shows the temporal evolution of the total energy $E_{\text {tot }}$ for different rotators that are ignited with the $c 3$ ignition. Note that the more massive WDs start from more negative values of total energy. This is caused by the larger gravitational energies $E_{\text {grav }}$ for heavier stars (see Table 4). All simulations end in explosions that cause the star to unbind at $t \sim 1 \mathrm{~s}$. This takes longest for the heaviest WD, AWD3. The differences in $E_{\text {tot }}$ that are apparent in the beginning become smaller during the explosion process, which is caused by a higher amount of released nuclear energy $E_{\text {nuc }}$ for the more massive WDs.

The non-rotating star exhibits the highest value of $E_{\text {tot }}$ in the homologous expansion phase. $E_{\mathrm{tot}}$ at $t=5 \mathrm{~s}$ consists mainly of kinetic energy $E_{\text {kin }}$ of the ejecta for the typical temporal evolution of the energy contributions. Accordingly, the ejecta are fastest for the non-rotating progenitor on average.

With respect to the stellar composition after the explosion, the following trend is visible from Table 4 and Fig. 6(b): the heavier the progenitor, the more fuel is left unburnt. The amount of IGEs after $5 \mathrm{~s}$ ranges from $0.54 M_{\odot}$ for the non-rotating star 


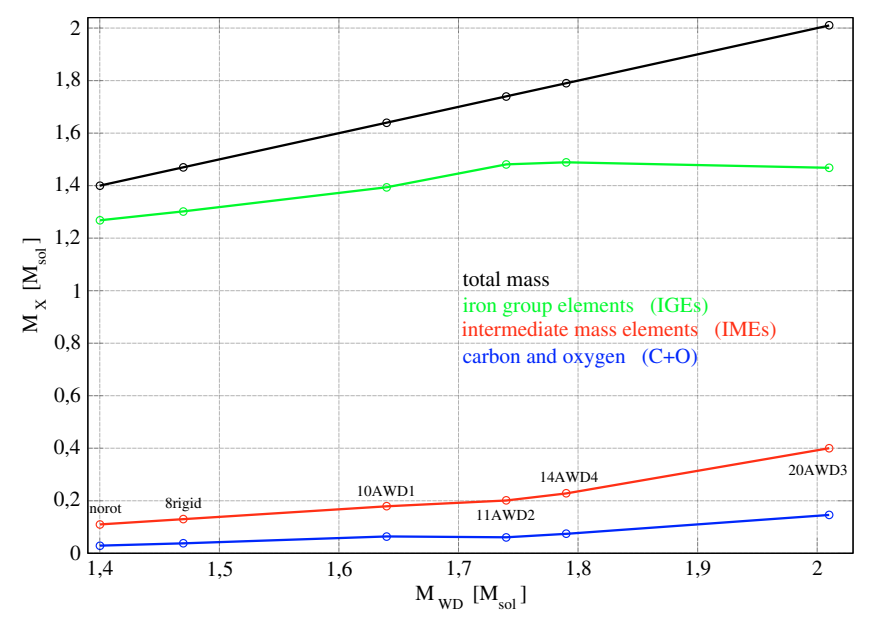

(a) no expansion: absolute fractions

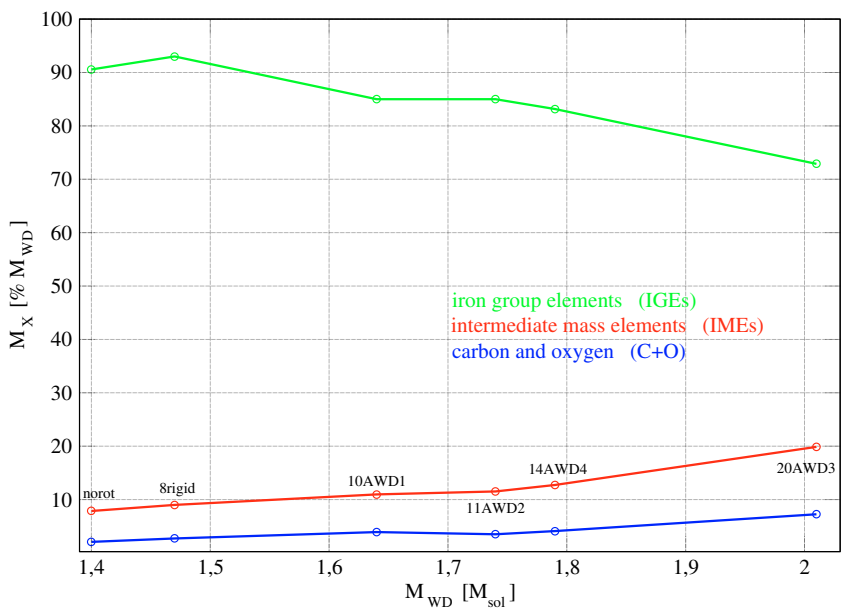

(b) no expansion: relative fractions

Fig. 5. Estimate of the burning products if the stars were not expanding during the burning process. The figure shows the fractions of the total mass in the high density regime $\left(\rho>5.25 \times 10^{7} \mathrm{~g} / \mathrm{cm}^{3}\right.$, "IGEs"), the medium density regime $\left(5.25 \times 10^{7} \mathrm{~g} / \mathrm{cm}^{3}>\rho>1.05 \times 10^{7} \mathrm{~g} / \mathrm{cm}^{3}\right.$, "IMEs"), and the low density regime $\left(\rho \leq 1.05 \times 10^{7} \mathrm{~g} / \mathrm{cm}^{3}, " \mathrm{C}+\mathrm{O}\right.$ ").

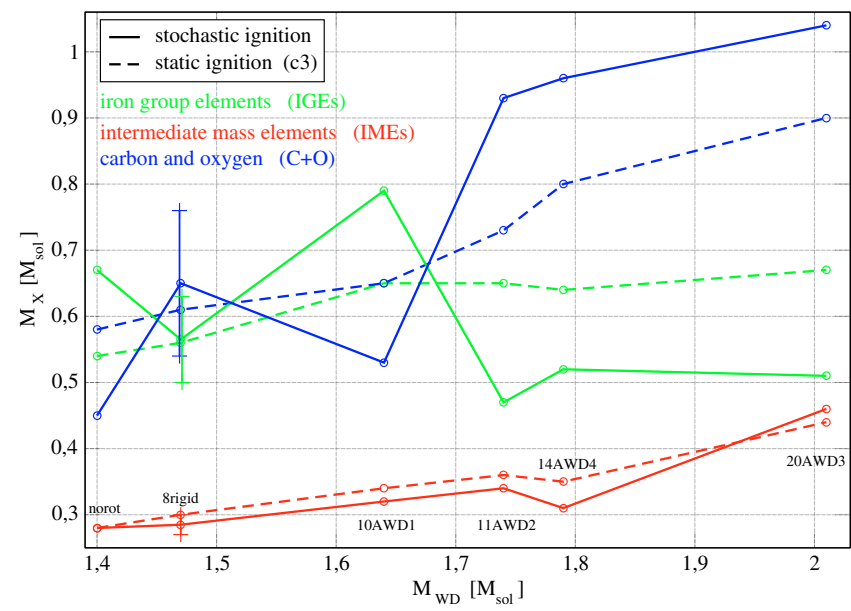

(a) pure deflagration: absolute fractions

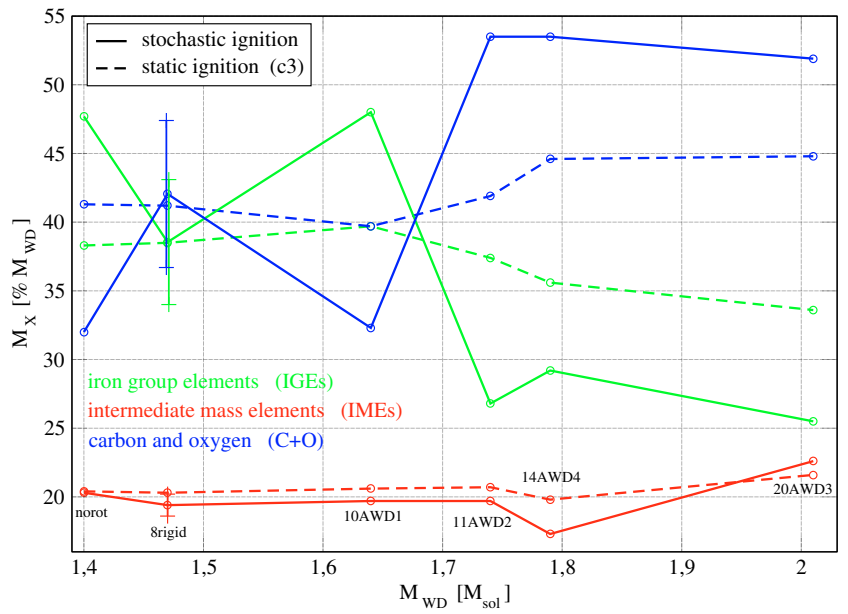

(b) pure deflagration: relative fractions

Fig. 6. Burning products after $t=5 \mathrm{~s}$ as the outcome of simulations that are ignited by stochastic ignition (solid lines) and $c 3$ ignition (dashed lines). Note the influence of the stochastic burning on the explosion outcome as can be seen for the rigid rotator: there the burning products are taken as the mean of two identical simulations that differ only in the random numbers for stochastic ignition.

to $0.67 M_{\odot}$ for the rapidly rotating model AWD3. However, the fraction of IGEs with respect to the total mass decreases for rapid rotation. Only $33 \%$ of the total $2.02 M_{\odot}$ is converted to IGEs for the AWD3 rotator. The amount of IMEs rises for more rapid rotation, its fraction is remarkably constant at $\sim 20 \%$ for all rotation laws. Combined with the fact that the amount of unburnt material becomes larger for faster rotators, this result indicates that the higher absolute amount of IMEs for the rapid rotators is not obtained "at the cost" of fuel in contrast to observations. It is a consequence of the small amount of produced IGEs.

\subsubsection{Stochastic ignition}

Basically, the trends found in the case of the $c 3$ ignition scenario remain valid for explosions with stochastic ignition. However, the energetics of the explosions show a larger variation (see Fig. 7(b) and Table 5), and even less IGEs are produced while more unburnt fuel is left over (see Figs. 6(a) and 6(b) as well as Table 5). This is explained by the fact that, unlike the symmetric $c 3$ ignition, the stochastically ignited bubbles are immediately subject to buoyant motion since the net buoyancy is not balanced by a symmetric alignment of the ashes, therefore having less time to burn the central region efficiently. Also fluctuations in the composition between different progenitor models are more distinct for stochastic ignition compared to $c 3$ ignition. This is related to the fact that not only the overall number of ignitions but also the specific locations of the ignitions influence the explosion outcome.

The most energetic explosions with high fractions of IGEs result from the non-rotating and the AWD1 progenitor models. For the latter model, the central angular velocity is low compared to the other differentially rotating models. Since convection in the pre-supernova phases causes efficient angular momentum transport throughout the core, however, the AWD1 
J. M. M. Pfannes et al.: Deflagrations of rapidly rotating white dwarfs. I.

Table 5. Energetics and compositions for the deflagration model activated by the stochastic ignition with $C_{\mathrm{e}}=5 \times 10^{3}\left(C_{\mathrm{e}}=5 \times 10^{4}\right.$ for AWD4) in different rotators.

\begin{tabular}{|c|c|c|c|c|c|c|}
\hline & Norot & Rigid / Rigid* & AWD1 & AWD2 & AWD4 & AWD3 \\
\hline$M_{\text {tot }}\left[M_{\odot}\right]$ & 1.40 & 1.47 & 1.64 & 1.74 & 1.79 & 2.02 \\
\hline \multicolumn{2}{|c|}{$C_{\mathrm{e}} 5 \times 10^{3}$} & $5 \times 10^{3}$ & $5 \times 10^{3}$ & $5 \times 10^{3}$ & $5 \times 10^{4}$ & $5 \times 10^{3}$ \\
\hline$\Omega[\pi]$ & 2 & 2 & 2 & 2 & 2 & 2 \\
\hline$I_{2 \pi}$ & 172 & $167 / 163$ & 168 & 154 & 404 & 152 \\
\hline \multicolumn{7}{|l|}{$t=0 \mathrm{~s}$} \\
\hline$E_{\text {grav }}\left[10^{51} \mathrm{erg}\right]$ & -3.027 & -3.204 & -3.665 & -4.027 & -4.135 & -4.518 \\
\hline$E_{\text {rot }}\left[10^{50} \mathrm{erg}\right]$ & 0.00 & 0.44 & 1.75 & 2.62 & 2.99 & 4.46 \\
\hline$\beta[\%]$ & 0.00 & 1.38 & 4.78 & 6.50 & 7.22 & 9.87 \\
\hline \multicolumn{7}{|l|}{$t=5 \mathrm{~s}$} \\
\hline$E_{\text {kin }}\left[10^{50} \mathrm{erg}\right]$ & 8.69 & $7.70 / 5.63$ & 8.64 & 3.67 & 3.68 & 3.61 \\
\hline$E_{\text {tot }}\left[10^{50} \mathrm{erg}\right]$ & 8.16 & $7.04 / 4.82$ & 7.84 & 1.95 & 1.97 & 1.33 \\
\hline$E_{\mathrm{nuc}}\left[10^{51} \mathrm{erg}\right]$ & 1.282 & 1.234 / 1.005 & 1.495 & 1.016 & 1.073 & 1.182 \\
\hline IGEs $\left[M_{\odot}\right]$ & 0.67 & $0.63 / 0.50$ & 0.79 & 0.47 & 0.52 & 0.51 \\
\hline$\left.M_{\mathrm{tot}}\right]$ & 48 & 43 / 34 & 48 & 27 & 29 & 25 \\
\hline IMEs $\left[M_{\odot}\right]$ & 0.28 & $0.30 / 0.27$ & 0.32 & 0.34 & 0.31 & 0.46 \\
\hline$\left[\% M_{\mathrm{tot}}\right]$ & 20 & $20 / 19$ & 20 & 20 & 17 & 23 \\
\hline $\mathrm{C}+\mathrm{O}\left[M_{\odot}\right]$ & 0.45 & $0.54 / 0.70$ & 0.53 & 0.93 & 0.96 & 1.04 \\
\hline$\left[\begin{array}{ll}\% & M_{\mathrm{tot}}\end{array}\right]$ & 32 & $37 / 47$ & 32 & 53 & 54 & 52 \\
\hline
\end{tabular}

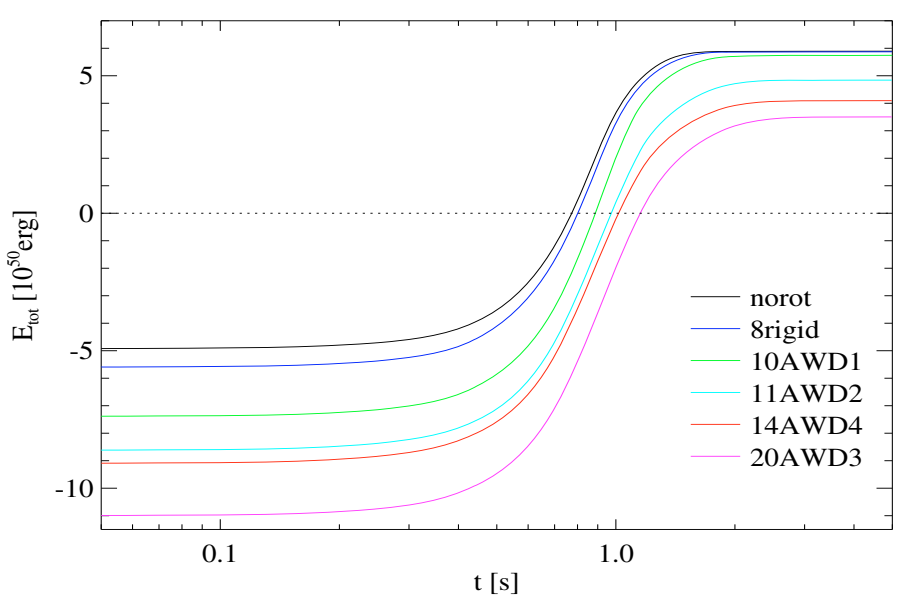

(a) rotation law: $c 3$ ignition

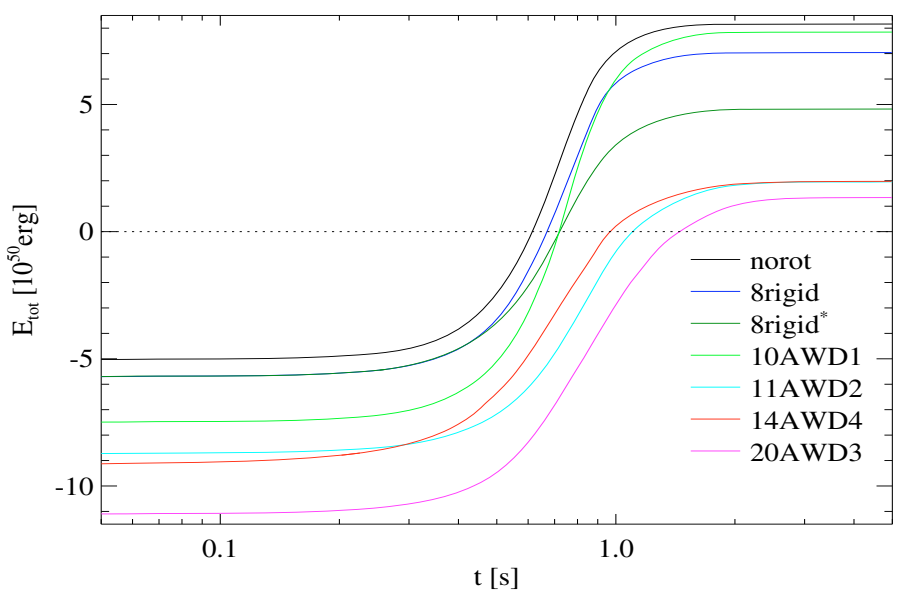

(b) rotation law: stochastic ignition

Fig. 7. Temporal evolution of the total energy for the deflagration study that is initiated by $c 3$ ignition in different rotators $\mathbf{a})$, and by stochastic ignition in different rotators $\left(C_{\mathrm{e}}=5 \times 10^{4}\right.$ for the AWD4 rotator, else $\left.\left.C_{\mathrm{e}}=5 \times 10^{3}\right) \mathbf{b}\right)$.
Table 6. Energetics and compositions for the deflagration model activated by stochastic ignition with $C_{\mathrm{e}}=5 \times 10^{3}$ in the $j_{\text {const }}$ and $v_{\text {const }}$ rotators.

\begin{tabular}{rcc}
\hline \hline & $j_{\text {const }}$ & $v_{\text {const }}$ \\
\hline$M_{\text {tot }}\left[M_{\odot}\right]$ & 1.80 & 1.71 \\
$C_{\mathrm{e}}$ & $5 \times 10^{3}$ & $5 \times 10^{3}$ \\
$\Omega[\pi]$ & 2 & 2 \\
$I_{2 \pi}$ & 159 & 158 \\
\hline$t=0 \mathrm{~s}$ & & \\
$E_{\text {grav }}\left[10^{51} \mathrm{erg}\right]$ & -4.271 & -4.056 \\
$E_{\text {rot }}\left[10^{50} \mathrm{erg}\right]$ & 3.16 & 2.39 \\
$\beta[\%]$ & 7.40 & 5.89 \\
\hline$t=5 \mathrm{~s}$ & & \\
$E_{\text {kin }}\left[10^{50} \mathrm{erg}\right]$ & 1.66 & 1.29 \\
$E_{\text {tot }}\left[10^{50} \mathrm{erg}\right]$ & -0.75 & -1.11 \\
$E_{\text {nuc }}\left[10^{51} \mathrm{erg}\right]$ & 0.817 & 0.679 \\
$\mathrm{IGEs}\left[M_{\odot}\right]$ & 0.34 & 0.29 \\
{$\left[\% M_{\text {tot }}\right]$} & 19 & 17 \\
$\mathrm{IMEs}\left[M_{\odot}\right]$ & 0.34 & 0.27 \\
{$\left[\% M_{\text {tot }}\right]$} & 19 & 16 \\
$\mathrm{C}+\mathrm{O}\left[M_{\odot}\right]$ & 1.12 & 1.15 \\
{$\left[\% M_{\text {tot }}\right]$} & 62 & 67 \\
\hline
\end{tabular}

rotator appears to be a rather unlikely outcome of an evolutionary scenario leading to a thermonuclear supernova. For the "AWD4 $C_{\mathrm{e}}=5 \times 10^{4}$ " explosion model, on the other hand, the amount of IMEs is somewhat higher, but this is compensated by little production of IGEs. Consequently, a larger fraction of fuel remains unburnt. The " $j_{\text {const }} C_{\mathrm{e}}=5 \times 10^{3}$ " and " $v_{\text {const }}$ $C_{\mathrm{e}}=5 \times 10^{3 \text { " }}$ explosion models generate yet smaller amounts of IGEs (see Table 6). However, neither the $j_{\text {const }}$ nor the $v_{\text {const }}$ rotation law are likely to occur in WDs. For the critically rotating rigid rotator, on the other hand, approximately the same results are obtained as in the case of the non-rotating star. Consequently, this type of rotating WD appears to be a progenitor candidate for type Ia supernovae caused by pure deflagrations. 


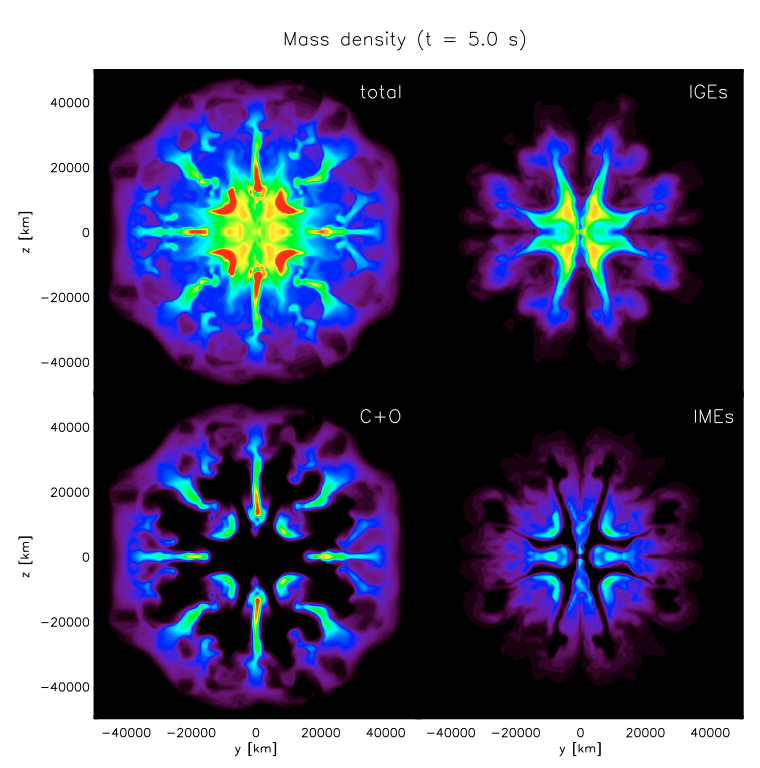

(a) "norot $c 3$ "explosion model
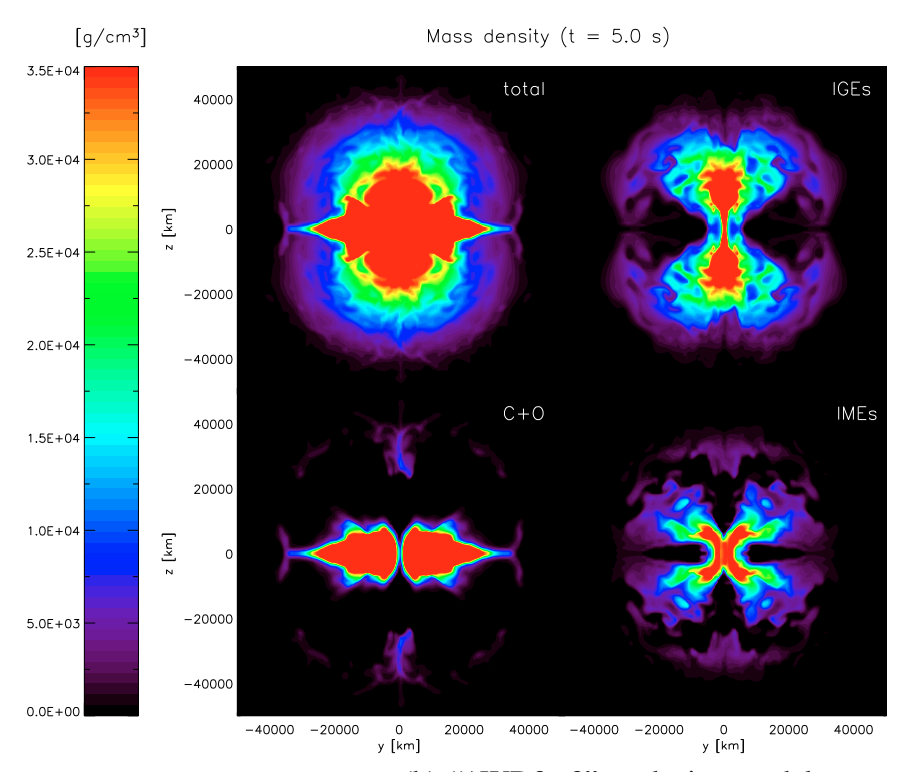

(b) "AWD3 c3" explosion model

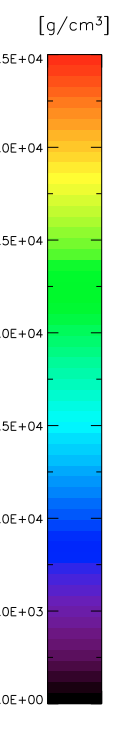

Fig. 8. Total (upper left) and fractional (upper right: IGEs; lower left: unburnt fuel; lower right: IMEs) mass densities after $t=5 \mathrm{~s}$ for the "norot c3" a) and "AWD3 $c 3$ " b) explosion models. A mixing of the species throughout the star emerges in both scenarios, particularly the rapid rotator leaves fuel in the stellar core. Cross sections along the rotation axis of simulations exhibiting equatorial symmetry are shown.

\subsection{Shear motion during the explosion}

It is a common belief that the shear introduced by differential rotation can enhance the explosion strength by increasing the flame surface. Although rotating WDs exhibit high rotation velocities (e.g., the fastest regions of the AWD1 rotator move with $10 \%$ of the speed of light), within the entire span of time during which burning takes place $(t \lesssim 1.5 \mathrm{~s}$ ) the direct influence of rotation on the flame surface is limited. The rotators accomplish only about half a rotation if the burning induced expansion is neglected. The latter arranges a slowdown of the star as soon as the thermonuclear runaway is initiated as a result of angular momentum conservation.

Unless a distinct jump in $\Omega$ occurs - which is not the case for smooth differential rotation -, the flame will not be significantly influenced by rotation in a direct way (but, as described in Sect. 5.1, by buoyancy and angular momentum effects).

Yoon \& Langer (2004) proposed that a rotation could trigger a deflagration-to-detonation transition since the outer layers still rotate rapidly when the outward burning flame causes a slowdown of the WD. The resulting gradient in velocity due to burning is higher than by differential rotation and could possibly generate a detonation if the flame surface is increased abruptly. In particular, such a rapid jump of angular velocity is found for the rotation law of a WD merger (Yoon et al. 2007). We do not consider DDTs in this work; however, an investigation of shear-induced DDTs with the method introduced by Golombek $\&$ Niemeyer (2005) would be possible and interesting. In particular, if observational evidence for rapid rotation of the progenitor star should be found, the DDT mechanism will be favored since prompt detonations of rapid rotators cannot explain normal SNe Ia (Pfannes et al. 2010).

\subsection{Expected spectral features}

An inspection of the composition and the kinematics of the ejecta in the homologous expansion phase allows a prediction of spectral features even without employing a detailed postprocessing study. Regarding rotation, the most serious discrepancy between the simulation results and observations is the prediction of $\mathrm{C}$ and $\mathrm{O}$ at low radial expansion velocities, i.e., close to the centre. This result, already a problem for the non-rotating deflagration model, grows more acute for rapid rotation. Figure 8 shows the total and fractional mass densities after $t=5 \mathrm{~s}$ for the "norot $c 3$ " and "AWD3 c3" explosion models. For the sake of demonstration, only the $c 3$ ignition is presented here. The results are qualitatively in accordance with those of the stochastic ignition.

SN Ia observations indicate that the IMEs should be located in the outer regions and enclose the IGEs for normal SNe Ia, whereas $\mathrm{C}$ and $\mathrm{O}$ must not be present near the centre at all. However, as a result of the turbulent combustion whose driving force is the Rayleigh-Taylor instability, the species are mixed throughout the stellar interior. Furthermore, the existence of a significant amount of unburnt fuel in the core is inconsistent with observations. The following trend that becomes stronger for more rapid rotation emerges: Initially, burning mostly advances towards the stellar poles and generates IGEs. Later, when the flame spreads toward the equatorial plane and the density has already dropped, IMEs are produced. However, the burning process ceases before the central part and material close to the equatorial plane can be burnt. This is reflected by the distinct peak of $\mathrm{C}$ and $\mathrm{O}$ at low radial velocities that can be seen in Fig. 9 for the AWD4 rotator. For comparison, the distributions in radial velocity space are also plotted for the non-rotating stochastic ignition model.

The highest radial velocities in the non-rotating case are $\lesssim 15 \times 10^{8} \mathrm{~cm} / \mathrm{s}$. Consequently, high velocity features cannot be explained by the deflagration model, although higher velocities might become apparent with increasing resolution. In the rotating case, the radial velocities are even smaller $\left(\lesssim 10 \times 10^{8} \mathrm{~cm} / \mathrm{s}\right)$, despite the fact that rotating progenitors include a large amount of rotational kinetic energy. This is due to the greater gravitational attraction of the rotating stars (cf. Table 3), and in 
J. M. M. Pfannes et al.: Deflagrations of rapidly rotating white dwarfs. I.

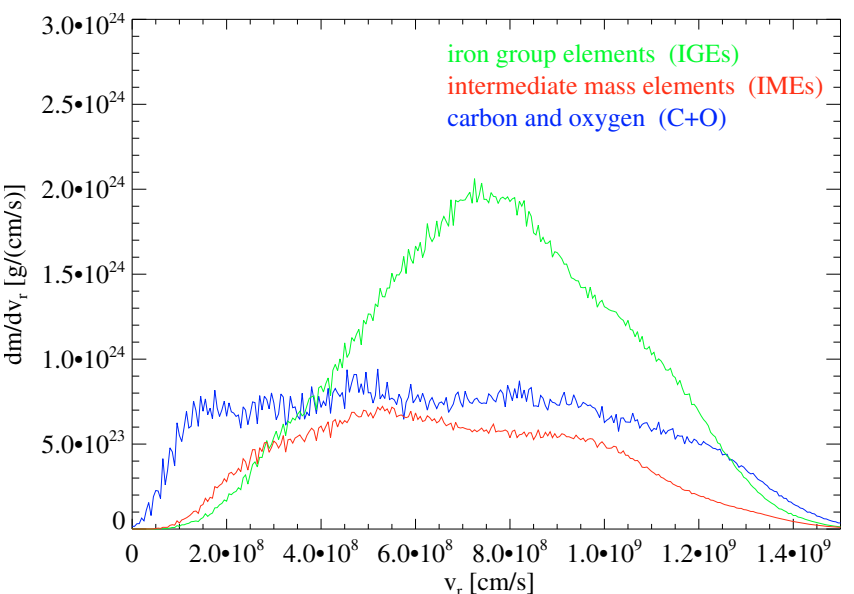

(a) "norot $C_{\mathrm{e}}=5 \times 10^{4}$ "

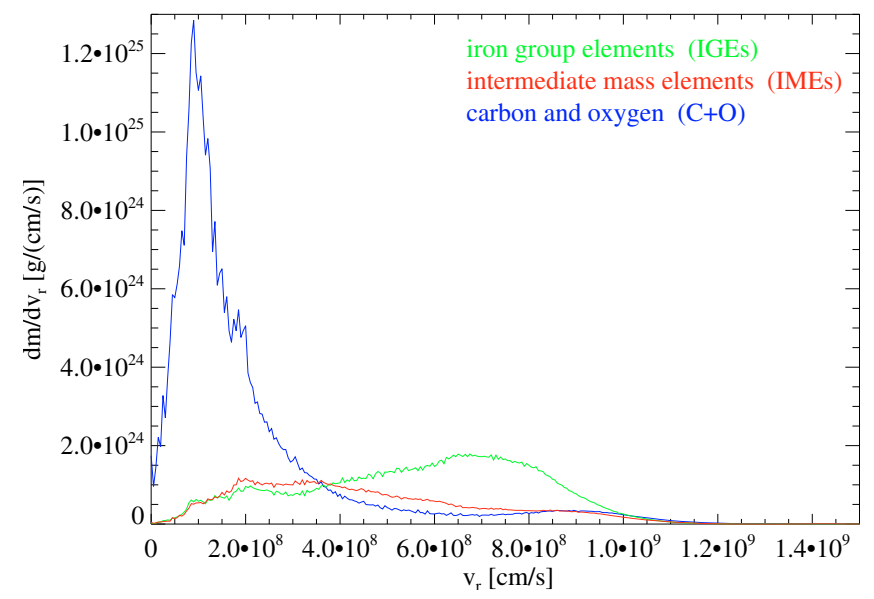

(b) "AWD4 $C_{\mathrm{e}}=5 \times 10^{4}$ "

Fig. 9. Probability density functions in radial velocity space for the "norot $C_{\mathrm{e}}=5 \times 10^{4}$ " a) and "AWD4 $C_{\mathrm{e}}=5 \times 10^{4}$ " b) explosion models.

addition the released energy is comparatively small because of incomplete burning. Figure 10 compares the initially available rotational energy (bottom of the bars) and the kinetic energies of the ejecta after $t=5 \mathrm{~s}$ (top of the bars) for different WD models. Except for the AWD1 rotator without convective core, the disposable kinetic energies become successively smaller for increasing strength of rotation. Note that for the "AWD3 $C_{\mathrm{e}}=$ $5 \times 10^{3}$ " explosion model, the kinetic energy after $t=5 \mathrm{~s}$ is even less than the kinetic energy initially present in the rotation motion.

\section{Conclusions}

In this paper, we investigated the influence of nearly critical rotation of the progenitor star on SN Ia explosions. We performed three dimensional numerical simulations of thermonuclear deflagrations for different rotation laws of the white dwarf progenitors and various initial conditions. The influence of the ignition process turns out to be larger in rotating stars compared to their non-rotating counterparts, which might contribute to the observed diversity among SNe Ia.

The main result of this study is that the amount of iron group elements is not significantly increased compared to the non-rotating case, although the rotating WD models are notably

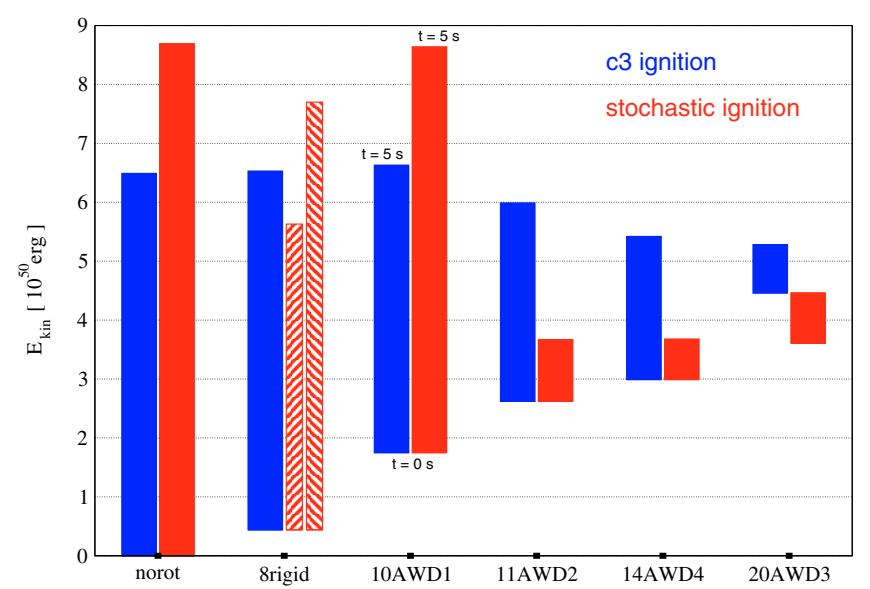

Fig. 10. Initially available rotational energy (bottom of the bars) and kinetic energy of the ejecta after $t=5 \mathrm{~s}$ (top of the bars) for successively heavier WD models.

heavier than their non-rotating counterparts. Due to the centrifugal expansion, rotators contain more material at low densities in general, thus the amount of material capable of burning to iron group elements is only weakly increased if the star is burnt by a deflagration. Furthermore, due to rotationally induced anisotropic buoyancy effects and, at the same time, inhibition of effective mixing parallel to the equatorial plane, the flame preferentially propagates toward the stellar poles. This leads to comparably weak and anisotropic explosions that leave behind unburnt material at the centre and in the equatorial plane. Moreover, no significant effects of shear motion acting on the flame are observed. Deflagrations of rapid rotators do not cause high velocity features but result in overall low expansion velocities. As a consequence, the pure deflagration scenario is ruled out in the case of rapid rotation of the progenitor star. The incineration of the critical rigid rotator shows similar features as the non-rotating scenario. Therefore, deflagrations could be possible for critical rigid rotation.

In conclusion, rotation of the progenitor star is unlikely to be the parameter that causes the observed variation in peak luminosities among SNe Ia. Rapid rotation of the progenitor star derived by an accretion study can lead to a great variety in explosion strengths in the deflagration scenario, but only within a range that is ruled out for observational reasons. Otherwise, even the possible critical rigid rotation can not account for a significant spread in the explosion outcome.

Acknowledgements. We thank Ewald Müller, Friedrich Röpke and Sung-Chul Yoon for helpful comments and discussions.

\section{References}

Davies, R. M., \& Taylor, G. 1950, R. Soc. London Proc Ser. A, 200, 375 Eriguchi, Y., \& Müller, E. 1985, A\&A, 146, 260

Fryxell, B., Olson, K., Ricker, P., et al. 2000, ApJS, 131, 273

Glimm, J., \& Li, X. L. 1988, Physics of Fluids, 31, 2077

Golombek, I., \& Niemeyer, J. C. 2005, A\&A, 438, 611

Hillebrandt, W., \& Niemeyer, J. C. 2000, ARA\&A, 38, 191

Kuhlen, M., Woosley, S. E., \& Glatzmaier, G. A. 2006, ApJ, 640, 407

Livio, M. 2000, in type I Supernovae: Theory and Cosmology, ed. J. C. Niemeyer, \& J. W. Truran, 33

Müller, E., \& Eriguchi, Y. 1985, A\&A, 152, 325

Müller, E., \& Steinmetz, M. 1995, Comp. Phys. Commun., 89, 45

Niemeyer, J. C. 1999, ApJ, 523, L57

Niemeyer, J. C., \& Woosley, S. E. 1997, ApJ, 475, 740

Pfannes, J. M. M., Niemeyer, J. C., \& Schmidt, W. 2010, A\&A, 509, A75 
A\&A 509, A74 (2010)

Plewa, T., Calder, A. C., \& Lamb, D. Q. 2004, ApJ, 612, L37

Read, K. I. 1984, Physica D Nonlinear Phenomena, 12, 45

Reinecke, M. 2001, Ph.D. Thesis, MPA Garching

Reinecke, M., Hillebrandt, W., \& Niemeyer, J. C. 2002, A\&A, 386, 936

Riess, A. G., Strolger, L.-G., Casertano, S., et al. 2007, ApJ, 659, 98

Röpke, F. K. 2005, A\&A, 432, 969

Röpke, F. K. 2008, in Supernovae: lights in the darkness, Proceedings of Science [arXiv:0804.2147]

Röpke, F. K., \& Niemeyer, J. C. 2007, A\&A, 464, 683

Röpke, F. K., Hillebrandt, W., Niemeyer, J. C., et al. 2006, A\&A, 448, 1

Röpke, F. K., Hillebrandt, W., Schmidt, W., et al. 2007a, ApJ, 668, 1132
Röpke, F. K., Woosley, S. E., \& Hillebrandt, W. 2007b, ApJ, 660, 1344 Schmidt, W., \& Niemeyer, J. C. 2006, A\&A, 446, 627

Schmidt, W., Niemeyer, J. C., \& Hillebrandt, W. 2006a, A\&A, 450, 265

Schmidt, W., Niemeyer, J. C., Hillebrandt, W., et al. 2006b, A\&A, 450, 283

Steinmetz, M., Müller, E., \& Hillebrandt, W. 1992, A\&A, 254, 177

Wood-Vasey, W. M., Miknaitis, G., Stubbs, C. W., et al., R. 2007, ApJ, 666, 694

Woosley, S. E., Wunsch, S., \& Kuhlen, M. 2004, ApJ, 607, 921

Wunsch, S., \& Woosley, S. E. 2004, ApJ, 616, 1102

Yoon, S.-C., \& Langer, N. 2004, A\&A, 419, 623

Yoon, S.-C., \& Langer, N. 2005, A\&A, 435, 967

Yoon, S.-C., Podsiadlowski, P., \& Rosswog, S. 2007, MNRAS, 380, 933 